\title{
Adaptation, trahison: la 'Silvie' de Landois et la genèse du drame bourgeois
}

\section{Pierre Hartmann}

\section{(2) OpenEdition}

1 Journals

\section{Édition électronique}

URL : https://journals.openedition.org/studifrancesi/40152

DOI : 10.4000/studifrancesi.40152

ISSN : 2421-5856

Éditeur

Rosenberg \& Sellier

\section{Édition imprimée}

Date de publication : 1 juillet 2004

Pagination : 95-100

ISSN : 0039-2944

\section{Référence électronique}

Pierre Hartmann, « Adaptation, trahison: la 'Silvie' de Landois et la genèse du drame bourgeois », Studi Francesi [En ligne], 142 (XLVIII | I) | 2004, mis en ligne le 30 novembre 2015, consulté le 09 septembre 2021. URL : http://journals.openedition.org/studifrancesi/40152 ; DOI : https://doi.org/10.4000/ studifrancesi.40152

\section{(c) (i) (9)}

Studi Francesi è distribuita con Licenza Creative Commons Attribuzione - Non commerciale - Non opere derivate 4.0 Internazionale. 


\section{Adaptation, trahison: la 'Silvie' de Landois et la genèse du drame bourgeois}

On sait que durant toute la période classique et au-delà, le genre romanesque a servi de magasin d'idées aux auteurs dramatiques en quête de sujets inédits ou de situations extraordinaires. Le passage de la forme romanesque à la forme dramatique ne va pourtant pas de soi; au-delà des problèmes d'ordre technique qu'il pose à l'écrivain comme au dramaturge, il soulève des questions de fond, qui ressortissent au domaine de la poétique, et plus généralement de l'esthétique. On pressent que s'ouvre ici, au critique comme à l'historien de la littérature, un vaste champ de réflexion qui, pour avoir été arpenté à de nombreuses reprises, n'a pas encore fait l'objet d'une investigation systématique. Loin de prétendre entamer un travail d'une telle ampleur, je me contenterai d'examiner, dans ce modeste article, un moment presque minuscule de ce long dialogue entretenu par les auteurs dramatiques avec leurs confrères romanciers. Moment minuscule parce qu'il concerne une pièce sans valeur propre, mais non dépourvu d'intérêt parce qu'étroitement lié à la naissance d'un genre appelé à un grand avenir, le drame bourgeois. S'agissant de la genèse de ce genre novateur, on dispose en effet d'une petite pièce anonyme signalée jadis par l'historien du théâtre H. C. Lancaster comme «la première tragédie en prose du 18ème siècle à avoir été jouée à la Comédie française» ${ }^{1}$. Il s'agit de la Silvie, représentée en 1741 et attribuée à Landois par les Anecdotes dramatiques de La Porte 2 . Or cette pièce appartient précisément à la longue liste de celles qui ont puisé dans le répertoire romanesque, puisqu'elle tire son argument des Illustres françaises de Robert Challe, plus précisément de l'avant-dernière des sept nouvelles qui constituent ce recueil, l'Histoire de des Francs et de Silvie. Ainsi, dès l'origine, le drame a partie liée avec le roman, si bien qu'il m'a semblé à la fois intéressant et pertinent d'étudier les spécifications naissantes du genre non par le biais de sa différenciation explicite d'avec la tragédie, mais par le truchement de la réélaboration à laquelle il contraint son modèle romanesque

De par sa structure et en dépit de sa minceur, la pièce de Landois se prête à l'exercice. Elle est en effet composée de deux parties: le drame proprement dit, et le prologue en trois scènes qui se propose d'en établir la nouveauté esthétique et d'en discuter la validité. Ce prologue, lui-même conçu sous une forme dramatique, selon un usage déjà suivi par Marivaux dans L'Ile de la Raison (1727), confronte cinq personnages autour de quelques uns des thèmes majeurs de la nouvelle dramaturgie en quête de ses propres règles: emploi de la prose au lieu des vers, ton familier, vraisemblance de l'action et des caractères, présence d'accessoires etc. Cette discussion ne porte pas à proprement parler sur Silvie, mais s'engage à l'occasion de sa représenta-

(1) H. C. LANCASTER, The first french Tragedie bourgeoise. Silvie, Baltimore, The John Hopkins Press, 1954. Comte tenu du caractère fluctuant des dénominations, je parlerai indifféremment, dans le cours de cet article, de tragédie (en prose) ou de drame (bourgeois).

(2) Outre l'édition moderne procurée par H. C. Lancaster, on peut consulter le texte de la pièce à la bibliothèque de l'Arsenal, sous la cote Th.N. 39. 932. Quoique les Anecdotes dramatiques soient postérieures d'une trentaine d'années à la représentation de Silvie, son attribution à Paul Landois n'est pas contestée. Ce marginal de la République des lettres écrivit de nombreux articles pour l'En- cyclopédie, et a moins passé à la postérité comme l'auteur de Silvie que comme le destinataire de la fameuse lettre de Diderot où la critique reconnaît habituellement l'expression la plus complète du déterminisme de son auteur (lettre du 29 Juin 1756).

(3) Je reprends donc à mon compte la thèse proposée par F. M. WEIL, qui reconnaît «dans les Illustres françaises une source importante du drame bourgeois». «Du roman bourgeois au drame bourgeois». Romanistische Zeitschrift für Literaturgeschichte, Heidelberg, Carl Winter Universitätsverlag, $1977\left(n^{\circ} 4\right)$, p. 454. 
tion, qui doit avoir lieu de façon imminente: le dialogue se noue en effet, comme déjà chez Marivaux, au foyer du Théâtre français, où les personnages devisent en attendant le lever de rideau, si l'on veut bien me passer cet anachronisme. C'est dire que la discussion ne porte pas particulièrement sur les mérites ou les défauts de la pièce proposée (qui reste à découvrir), mais, de façon plus générale, sur la nouvelle dramaturgie qu'annonce son sous-titre: «tragédie, en prose, en un acte». Landois a en effet l'habileté, peut-être la lucidité, de disjoindre la question de la valeur de son ouvrage de celle de la pertinence de son projet dramatique («la pièce va se jouer; si elle est sifflée, elle l'aura sans doute mérité; les personnes éclairées blâmeront l'ouvrage, sans condamner le projet»), devançant en cela le jugement mesuré mais définitif de Diderot dans le second de ses Entretiens sur le fils naturel: (sa pièce) «suffit pour éveiller un homme de génie, mais il faut un autre ouvrage pour convertir un peuple».

Passons rapidement sur le prologue dialogué de Silvie, qui ne mentionne à aucun moment la source romanesque du drame, et qui véhicule tant une représentation délibérément caricaturale de la tragédie classique qu'un ensemble de propositions destinées à cerner la poétique du nouveau genre. Ces propositions n'ont d'autre intérêt que d'avoir été précocement formulées; elles se retrouveront par la suite, avec une toute autre ampleur, dans les écrits théoriques de Diderot ou dans l'Essai sur le genre dramatique sérieux de Beaumarchais'. À cet égard, l'habileté de l'auteur de Silvie consiste à conférer à ses idées sur le drame une forme et un ton eux-mêmes accordés au genre qu'il promeut, fond et forme concourant de la sorte tant à la défense théorique qu'à l'illustration pratique de ses thèses. Le spectateur du drame est ainsi mis en présence, dès le prologue, d'un échantillon plausible du public habituel du Thêâtre Français, devisant in situ comme il vient peut-être de le faire lui-même, le foyer du théâtre imposant astucieusement une façon d'unité de lieu non dépourvue d'un certain baroquisme, par l'effet de miroir qu'il lui tend en qualité de spectateur réel de la pièce. S'impose alors comme allant de soi le ton intermédiaire de la libre conversation, mi-érudite mi-mondaine, propice aux échanges entre professionnels du théâtre et amateurs éclairés, sans préjudice des saillies burlesques d'un personnage de rencontre, simple curieux auquel est dévolu une double fonction de repoussoir et de faire valoir. Comme on voit, le prologue de Silvie parait crédible et se lit agréablement. Suivons-en maintenant les acteurs, passons avec eux du foyer aux loges, et voyons ce que l'adaptation du roman peut nous apprendre sur les prémices du drame bourgeois.

Une précision liminaire s'impose d'emblée: la Silvie de Landois est une adaptation de la sixième des sept nouvelles dont la réunion forme le recueil des Illustres françaises. Dans le dispositif romanesque challien, si bien analysé par Michèle Weils, les nouvelles n'apparaissent pas comme des entités narratives closes et repliées sur elle-même, mais comme des formes ouvertes s'insérant dans une construction englobante, celle du roman dialogique. C'est ainsi que l'histoire de Des Francs et Silvie ne trouve son explication qu'en dehors du récit chargé de l'illustrer, dans le cadre de la septième et dernière nouvelle du recueil. La construction raffinée du roman challien ne pouvait trouver de répondant dans la pièce en un acte de Landois, qui néglige délibérément de tels effets de structure pour procéder à un aplatissement du textesource, dont il lui faut ignorer la subtile dialectique interne. Â l'issue d'un tel traitement, ce n'est plus le roman qui constitue à proprement parler le texte-source, mais l'Histoire de Des Francs et Silvie soustraite à son cadre romanesque et ramenée à une nouvelle tragique de facture classique. Paradoxalement, l'innovation dramatique

(4) À l'égard de Beaumarchais, je renvoie à l'ouvrage de B. DiDIER (Beaumarchais ou la passion du drame, PUF, 1994) ainsi qu'à mon article: «Le
Projet esthétique de Beaumarchais», Dix-buitième siècle $\mathrm{n}^{\circ} 27, \mathrm{PUF}, 1995$ (p. 439-451).

(5) M. WEIL, Challe romancier, Genève, Droz, 1991. 
visée par Landois procède d'une lecture régressive et mutilante du roman novateur de Challe. Je laisse ouverte la question de savoir s'il s'agit là du destin normal de toute adaptation dramatique d'un texte romanesque.

Quel que soit en effet le genre pratiqué par l'auteur, les conventions inhérentes à la forme théâtrale imposent le choix restrictif d'un moment de l'action: lecteur d'un récit, le dramaturge se voit dans la nécessité de pratiquer une coupe et de resserrer l'intrigue autour d'une séquence cruciale' . Sur ce point, la pièce de Landois se montre respectueuse des pratiques dramatiques héritées du classicisme et toujours en vigueur au XVIII ${ }^{\text {me }}$ siècle. Landois ne songe pas un instant à adopter le rythme propre au théâtre élisabéthain, par lequel il aurait pu échapper à la première des unités pour se conformer à la chronologie romanesque, bien au contraire: sa pièce opère dans le tissu narratif une coupure radicale, qui tend à resserrer autant que faire se peut toute l'action dramatique. Alors que le récit challien, même ramené à l'épisode conclusif de la vengeance, couvre une durée de plusieurs semaines, la pièce de Landois se déroule pour ainsi dire en temps réel: approximativement une heure de représentation pour une action qui peut plausiblement s'effectuer dans un laps de temps égal. À propos de cet aspect capital de l'écriture dramatique, la première tragédie en prose représentée ne propose donc nulle innovation. Se détournant du modèle shakespearien qui se verra si fréquemment sollicité par les futurs théoriciens du drame, Silvie n'anticipe pas davantage les choix prochains de la nouvelle dramaturgie allemande elle-même inspirée de Skakespeare, ni ceux d'un Rétif ou d'un Mercier. Marquons-le fortement: unité de temps, unité de lieu et unité d'action, les trois piliers de la dramaturgie classique ne sont nullement ébranlés par la tentative par ailleurs novatrice de Landois, alors même que le support romanesque de cette tentative invitait à une telle remise en cause $\mathrm{e}^{7}$. On pourrait en revanche être tenté de penser que le choix d'une action en temps réel va bien au-delà de la dramaturgie classique, et supputer qu'il ouvre au théâtre une voie qui ne sera frayée qu'à l'époque contemporaine. Mais il serait manifestement abusif de juger d'une telle singularité à cette aune: nullement théorisée dans le prologue, elle anticipe moins sur une dramaturgie de facture moderne qu'elle ne décèle la technique rudimentaire de son auteur.

Quant au moment de la diégèse romanesque retenu par le dramaturge, c'est celui de la vengeance. Rappelons-en rapidement les données factuelles: retour de voyage, le narrateur (Des Francs) surprend nuitamment sa femme dans les bras d'un ami répondant au patronyme de Gallouin. Maîtrisant à grand peine une impulsion meurtrière, il diffère sa vengeance, suscite un duel lors duquel il blesse gravement son rival, et séquestre son épouse à la campagne, en la réduisant cruellement au pain, à l'eau et aux haillons. C'est de cet épisode noir, le plus saisissant du roman, que le dramaturge s'empare, non sans lui imposer une compression drastique, puisque la première scène du drame nous découvre Des Francs au matin de la surprise, guettant le réveil de sa femme et donnant instruction à son laquais de transformer la chambre où il se tient en un sinistre lieu de réclusion:

Le thêâtre représente l'intérieur d'une chambre où l'on ne voit que les murs; une table sur laquelle est une lumière, un pot à l'eau et un pain, un habit d'homme et une mauvaise robe de chambre de femme.

La scénographie est astucieuse: habitué au faste décoratif du Théâtre Français, le spectateur ne peut qu'être surpris de se trouver face à un non-décor dont il compren-

(6) Lorsque le dramaturge se refuse à de telles contraintes, on aboutit à une œuvre monstreuse; témoin: le Drame de la vie de Rétif de la Bretonne.
(7) Il est vrai qu'alors, la pièce n'aurait pas été reçue aux Français... 
dra le motif à mesure qu'il progressera dans l'intelligence de l'intrigue, de conserve avec la victime. Landois semble ainsi souscrire par avance aux injonctions formulées par Diderot au dix-neuvième chapitre («De la décoration») de son essai De la poésie dramatique: «demandez seulement qu'on vous montre le lieu de la scène tel qu'il doit être». En l'occurrence, Landois ne se contente pas de déférer à cet impératif de bon sens, mais fait preuve d'une belle inventivité théâtrale. Le dénuement de l'espace scénique laisse en effet présager l'horreur du traitement réservé à l'héroïne, la scène devenant une sorte de miroir de l'action dramatique à venir (Silvie doit elle-même être dépouillée de ses vêtements, tondue etc.). Quoiqu'il en soit, les indications scéniques données par le dramaturge participent évidemment de cette volonté réformatrice condamnant le décor ad libitum et attentive au réalisme local, comme en témoigne encore l'importance conférée aux accessoires traditionnellement prohibées de la scène tragique. Il y a incontestablement, chez Landois, une part calculée de provocation.

La nouvelle de Challe repose sur un artifice d'intrigue: Silvie n'a trahi l'homme qu'elle aime que pour avoir été envoûtée à son insu par un procédé de type magique. Le tragique de la situation de l'héroïne réside alors dans l'insoluble énigme qui la désigne à ses propres yeux comme une coupable, sans qu'elle puisse s'expliquer à elle-même les motifs de sa trahison. Sur ce point, Landois modifie sensiblement les données de l'intrigue; chez lui, Silvie est simplement endormie par un narcotique. Probablement s'agit-il là d'une rationalisation conforme à l'évolution des esprits dans la première moitié du 18ème siècle; déjà le recours à un élément magique avait indisposé les premiers lecteurs de Challe, quelque trente années auparavant ${ }^{8}$. Mais cette modification s'avère lourde de conséquence: chez Landois, Silvie ne s'est ni endormie ni réveillée dans les bras de son séducteur; non seulement elle se croit innocente, mais elle ne comprend pas un traître mot aux imputations de son époux, qu'elle estime à bon droit calomnieuses. Sur cette base modifiée, Landois se devait d'écrire la véritable scène à faire de sa tragédie, la confrontation des deux époux qui correspond à celle de la dernière entrevue dans le roman', alors même que la visée du dramaturge diverge fondamentalement de celle du romancier. Dans la perspective bouchée de la nouvelle tragique, il s'agit pour Challe de faire la scène des adieux définitifs, à la manière, mutatis mutandis, de l'ultime entrevue de Nemours et de Mme de Clèves dans le roman phare de Mme de Lafayette. Dans la perspective optimiste du drame bourgeois, il s'agit en revanche de ménager la réconciliation des époux. Là où l'optique challienne impose la sobriété d'un récit pathétique mettant une ultime fois en présence deux amants contraints par la fatalité à renoncer l'un à l'autre, l'optique retenue par Landois débouche sur la frénésie d'un dialogue passionnel qui culmine dans la tentative d'un homicide réciproque. Dans l'avant-dernière scène du drame, quoiqu'encore convaincu de la culpabilité de sa femme, Des Francs se voit contraint de confesser la pérennité de ses sentiments, la frénésie meurtrière s'emparant alors des deux époux n'étant à tout prendre qu'une manifestation paroxystique du Liebestod. Dès lors, la voie est frayée pour le coup de théâtre conclusif.

En dépit de la nouveauté revendiquée par Landois, la scène finale de Silvie recourt aux poncifs d'une dramaturgie conventionnelle, associant au dénouement les catégories classiques de la révélation et du coup de théâtre. Le coup de théâtre, c'est l'entrée en scène d'un ami du couple, qui sauve les époux sur le point de s'entre-tuer; quant à la révélation, elle s'effectue sous la forme d'une confession épistolaire du séducteur mourant, attestant l'innocence de l'héroïne et, mieux encore, son

(8) Je renvoie au compte-rendu du Journal littéraire de La Haye, numéro de mai-juin 1713, dont on peut lire de larges extraits dans l'édition des Illustres françaises procurée par F. DELOFFrE et
J. Cormier pour le compte des éditions Droz (Genève, 1991, appendice $\mathrm{n}^{\circ} 1$, pp. 591-594).

(9) pp. 420-423 pour l'édition du roman susmentionnée, et septième scène du drame. 
intégrité physique: «la femme de chambre de Silvie m'avait livré sa maîtresse par le moyen d'un breuvage qui l'avait endormie» et «votre retour m'a empêché de consommer mon crime»! Dans le roman de Challe, l'adultère est dûment consommé, et le corps resplendissant de l'héroïne a été «souillée», selon la vigoureuse expression de l'auteur. Les modifications apportées par Landois altèrent sensiblement le sens de la fable challienne. En regard de la nouvelle tragique, qui charrie des éléments narratifs qui marqueront définitivement la conscience des protagonistes, la tragédie bourgeoise paraît une simple péripétie dramatique, une brève incursion du haut ton dans la prose de la vie quotidienne, une parenthèse ouverte par méprise dans une vie conjugale sans épaisseur ni mystère, et destinée, de ce fait à être promptement refermée. A vrai dire, il n'y a plus de drame, mais un simple malentendu; ou, selon les propres termes de Silvie, une «illusion» rapidement dissipée. Loin d'exprimer le caractère effroyable qu'elle possédait dans l'ancienne tragédie, mais loin aussi de revêtir l'aspect profondément affligeant qu'elle avait dans le roman, la révélation conclusive assume une fonction pacifiante: le conflit n'est pas tranché dans le sang et les larmes, mais résolu dans l'amour et par la réconciliation des antagonistes. Contrairement à son modèle, la tragédie en prose de Landois reçoit une conclusion heureuse, sans résidu ni scorie. Elle se rapproche ainsi du modèle de la comédie ou de l'ancienne tragi-comédie, dont les obstacles et les alarmes finissent par s'évanouir sans laisser de traces. En ce sens, Landois fait effectivement évoluer le théâtre de son temps en direction d'un genre intermédiaire, préparant les routes de Sedaine et de Diderot. L'appellation drame bourgeois conviendrait bien plus à son oeuvre que celle de tragédie, fût-elle éminemment prosaïque.

Du roman à la tragédie, il n'y a pas seulement affaissement du tragique, mais encore affaissement du message. Si la substitution d'un breuvage narcotique à un philtre magique marque un progrès dans la rationalité de l'intrigue, elle n'en oblitère pas moins la strate la plus profonde de la réflexion challienne. Contrairement au narcotique, le philtre implique la conscience et, en quelque façon, la participation affective de l'héroïne à l'entreprise de son séducteur. Il est alors loisible de se demander si le très rationaliste auteur des Difficultés sur la religion proposées au père Malebranche ne s'est pas servi de cet artifice pour traiter de façon oblique et recevable les motifs croisés de la séduction et du désir féminin, voire, plus précisément, le thème de l'opacité du désir et de sa relative autonomie à l'égard de la sphère sentimentale. L'ignorance de Silvie quant aux moyens utilisés par son séducteur la conduit en effet à une réflexion abyssale sur son être moral, sur la nature de son désir, sur l'énigmatique indifférence de son inclination non seulement à l'endroit des normes morales qu'elle reconnaît pour siennes, mais, chose plus troublante encore, à l'égard des motions sentimentales qui l'animent en faveur de son époux. A la faveur de l'artifice magique, la nouvelle challienne déploie en conséquence tout un arrière-plan psychologique d'une grande richesse, là où la dramaturgie rapide et carrée de Landois procède à une simplification radicale des données du problème, rabattu sur un lieu commun de la morale, celui de l'innocence calomniée. De cette déperdition de sens, sans doute Landois ne porte-t-il pas l'entière responsabilité. Marivaux excepté, quel dramaturge du 18 ème siècle peut-il se targuer de proposer une analyse psychologique à la hauteur de celle pratiquée par les romanciers contemporains? Quoiqu'il en soit, par le fait de donner le pas à l'enquête factuelle sur l'investigation psychologique et, simultanément, de privilégier la gesticulation dramatique relativement à l'antagonisme tragique, les choix simplificateurs opérés par Landois dessinent une trajectoire dont l'inflexion n'est pas étrangère à l'évolution du théâtre en direction du drame bourgeois en voie de constitution, et dont Silvie offre en effet une première approximation.

Au terme de cette étude, il importe de marquer nettement les limites de l'exercice. La pièce de Landois est de toute évidence une oeuvre trop mince pour qu'il paraisse possible d'en tirer des conclusions générales. Une mise en balance des possi- 
bilités respectives du roman et du théâtre à un moment donné de l'histoire littéraire impliquerait, pour faire sens, le choix d'un romancier et d'un dramaturge à parité de talent. De toute évidence, Landois n'est pas un Challe en cothurnes... Cependant, tel qu'il s'offre à nous dans sa médiocrité, mais aussi dans sa singularité et son indéniable précocité, l'opuscule de Landois ne laisse pas d'être riche d'enseignements. Diderot ne s'y est pas trompé, qui cite à deux reprises la tentative de son collaborateur, dont il n'hésite pas à faire, en l'occurrence, un précurseur. Que Silvie indique à la nouvelle dramaturgie en gestation au mitan du siècle des directions à suivre, cela n'est guère douteux: comment Diderot n'aurait-il pas été sensible à la sorte de pantomime qui en introduit l'action, et dont il n'est pas impossible qu'il se soit souvenu pour l'ouverture du Fils naturel? Outre cela, il n'est sans doute pas fortuit que cette première tentative ait été menée comme une adaptation romanesque: dans la volonté de créer une nouvelle dramaturgie, adaptée aux temps présents, a probablement compté la conscience diffuse d'une certaine avancée du genre romanesque. Le drame bourgeois emprunte au roman, conformément à une vieille tradition certes, mais aussi, plausiblement, en raison du retard enregistré dans l'expression dramatique sérieuse sur une forme qui a su se mettre plutôt qu'elle en phase avec son siècle. Aussi bien le choix du modèle paraitt-il judicieux: premier grand roman bourgeois sérieux, les Illustres françaises avaient naturellement vocation à retenir l'attention d'un écrivain soucieux de faire évoluer l'écriture dramatique en direction de ce qui deviendrait le drame bourgeois. Que cette adaptation soit simultanément une trahison ne fait alors que confirmer l'importance du retard, avec tout à la fois la difficulté et l'urgence qu'il y avait à tenter de le combler. 